\title{
Comparison between the luminosity functions of X-ray and [OIII] selected AGN
}

\author{
I. Georgantopoulos and A. Akylas
}

\begin{abstract}
Institute of Astronomy \& Astrophysics, National Observatory of Athens, Palaia Penteli, 15236, Athens, Greece e-mail: ig@astro.noa.gr
\end{abstract}

Received 21 July 2009 / Accepted 23 October 2009

\section{ABSTRACT}

\begin{abstract}
We investigate claims according to which the X-ray selection of AGN is not as efficient compared to that based on [OIII] selection because of the effects of X-ray absorption. We construct the predicted X-ray luminosity function both for all Seyferts as well as separately for Seyfert-1 and Seyfert-2 type galaxies, by combining the optical AGN [OIII] luminosity functions computed in SDSS with the corresponding $L_{\mathrm{X}}-L_{[\mathrm{OIII}]}$ relations. These relations are derived from XMM-Newton observations of all Seyfert galaxies in the Palomar spectroscopic sample of nearby galaxies after a correction for X-ray absorption and optical reddening. We compare the predicted X-ray luminosity functions with those actually observed in the local Universe by HEAO-1, RXTE as well as INTEGRAL. The last luminosity function is obtained in the $17-60 \mathrm{keV}$ region and thus is not affected by absorption even in the case of Compton-thick sources. In the common luminosity regions, the optically and X-ray selected Seyfert galaxies show reasonable agreement. We thus find no evidence that the $[\mathrm{OIII}]$ selection provides a more robust tracer of powerful AGN compared to the X-ray. Still, the optical selection probes less luminous Seyferts compared to the current X-ray surveys. These low luminosity levels, are populated by a large number of X-ray unobscured Seyfert-2 galaxies.
\end{abstract}

Key words. X-rays: general - X-rays: diffuse background - X-rays: galaxies

\section{Introduction}

Since the detection of the nearby AGN 3C 273 in X-rays by UHURU (Kellogg et al. 1971), X-ray observations have been considered to be the primary tool for selecting AGN. Recently, the deepest ever observations in the Chandra Deep Field North and South (Alexander et al. 2003; Giaconni et al. 2002; Luo et al. 2008) have resolved $80-90 \%$ of the extragalactic X-ray light, the $\mathrm{X}$-ray background, in the $2-10 \mathrm{keV}$ band. These observations reveal a sky density of about 5000 sources per square degree (Bauer et al. 2004), the vast majority of which are AGN (for a review see Brandt \& Hasinger 2005). These surveys made it possible to derive the luminosity function and probed with good accuracy the accretion history of the Universe (Ueda et al. 2003; La Franca et al. 2005; Barger et al. 2005).

In the local Universe, the X-ray luminosity function has been determined from the wide-angle surveys of RXTE and HEAO1. Sazonov \& Revnitsev (2004) derived the luminosity function for bright AGN, detected at fluxes $>2.5 \times 10^{-11} \mathrm{erg} \mathrm{cm}^{-2} \mathrm{~s}^{-1}$ in the 3-20 keV band, in the RXTE slew survey. Shinozaki et al. (2006) derived the luminosity function of AGN using the allsky HEAO-1 in the 2-10 keV band. Even these hard X-ray surveys may be missing a number of extremely obscured AGN. In the case of Compton-thick AGN, at column densities $>10^{24} \mathrm{~cm}^{-2}$ (equivalent to $A_{\mathrm{V}} \sim 450$ using the Galactic dust-togas ratio), a large fraction of the intrinsic flux will be absorbed. The SWIFT (Gehrels et al. 2004) and the INTEGRAL missions (Winkler et al. 2003), which carry ultra-hard X-ray detectors $(>15 \mathrm{keV})$ albeit with limited imaging capabilities probed energies which are immune to X-ray obscuration up to column densities of $10^{25} \mathrm{~cm}^{-2}$. These missions helped towards further constraining the number density of such heavily absorbed sources at very bright fluxes, $f_{17-60 \mathrm{keV}}>10^{-11} \mathrm{erg} \mathrm{cm}^{-2} \mathrm{~s}^{-1}$ in the local Universe, $z<0.1$ (Beckmann et al. 2006; Bassani et al. 2006; Winter et al. 2008; Winter et al. 2009). The luminosity function in these energies has been calculated by Sazonov et al. (2007), Paltani et al. (2008) and Tueller et al. (2009). It appears that the fraction of Compton-thick sources is small, and thus the RXTE and HEAO-1 luminosity functions are little affected.

In the optical, QSOs have been traditionally selected using colours (Schmidt \& Green 1983; Marshall et al. 1987; Boyle et al. 2000). The advent of the $2 \mathrm{dF}$ (Croom et al. 2004) and the SDSS (York et al. 2000) surveys have generated vast samples of QSOs providing a leap forward in the study of their luminosity function. The derived sky density of QSOs (few hundred per square degree) is at least an order of magnitude lower than the one derived from X-ray surveys. This is because the colour selection of AGN requires that the nuclear optical luminosity is much higher than that of the host galaxy for the AGN to be detected (typically $M_{B}<-23$ ). Therefore the optical selection based on colours is biased against low luminosity AGN in contrast to the $\mathrm{X}$-ray selection.

The limitations of colour optical selection techniques can be circumvented by selecting AGN via their emission lines. Such methods can extend the optical luminosity function to low luminosities. Ho et al. (1997) carried out a spectroscopic survey of about 500 nearby galaxies selected from the revised ShapleyAmes catalogue (Sandage \& Tammann 1981). They identified Seyfert emission-line characteristics in 52 galaxies. Ulvestad $\&$ Ho (2001) calculated the optical $B$-band luminosity function from their sample extending the AGN luminosity function to $M_{B} \sim-17$ (see also Georgantopoulos et al. 1999). A limitation in the above works is that the $B$-band is strongly affected by the host galaxy light. Hao et al. (2005a) extended these 
Table 1. $\operatorname{LogX}$-ray vs. $\log [\mathrm{OIII}]$ luminosity relation in optically selected AGN.

\begin{tabular}{lccccc}
\hline \hline Sample & Number & Slope & Intercept & $\left\langle\log \left(L_{\mathrm{x}} / L_{[\mathrm{OIII}]}\right)\right\rangle$ & Dispersion \\
\hline A. Seyfert-1 (Ho \& Heckman) & 28 & $0.84 \pm 0.09$ & $8.27 \pm 3.87$ & 1.66 & 0.60 \\
B. Seyfert-1 (Ho) & 8 & $0.66 \pm 0.22$ & $15.31 \pm 8.84$ & 1.84 & 0.82 \\
C. Seyfert-1 (Heckman) & 20 & $1.04 \pm 0.17$ & $-0.17 \pm 7.34$ & 1.59 & 0.48 \\
D. Seyfert-2 (Ho luminous+weak) & 23 & $0.83 \pm 0.15$ & $7.3 \pm 6.1$ & 0.87 & 0.80 \\
E. Seyfert-2 (Ho luminous) & 11 & $0.64 \pm 0.17$ & $15.34 \pm 7.15$ & 1.16 & 0.77 \\
F. Seyfert-2 (Ho weak) & 12 & $0.59 \pm 0.29$ & $16.18 \pm 11.48$ & 0.61 & 0.78 \\
G. Seyfert 1 + 2 (luminous) & 17 & $0.65 \pm 0.14$ & $15.35 \pm 5.90$ & 1.44 & 0.84 \\
\hline
\end{tabular}

results significantly, using the SDSS survey to select a sample of about $3000 \mathrm{AGN}$ in the redshift range $0<z<0.15$. Hao et al. (2005b) derive the Ha and [OIII] $\lambda 5007$ luminosity function separately for Seyfert-1 and Seyfert-2 galaxies. The [OIII] line is considered a much better proxy of the nuclear power as compared to the $B$-band luminosity, although in the case of strong star-forming emission some contamination of the [OIII] emission is expected.

The question which arises is whether the optical emission lines or the X-ray selection methods are more efficient for finding low-luminosity AGN. This can be addressed by comparing the X-ray and optical AGN luminosity functions. Heckman et al. (2005) addressed this issue by converting the optical [OIII] Seyfert (combined type 1 and type 2) luminosity function of Hao et al. (2005b) to X-ray wavelengths using the relation between $\mathrm{X}$-ray and [OIII] luminosity. Comparison with the RXTE X-ray luminosity function reveals that the latter lies consistently below the optical one. As these authors have intentionally neglected the $\mathrm{X}$-ray absorption, they conclude that the mismatch between the two luminosity functions can be more naturally attributed to absorption in X-ray wavelengths. The overall conclusion from this work is that the optical [OIII] emission may provide a more efficient method for picking AGN. In this paper we attempt to further address this issue and to understand the reason for the disagreement found by Heckman et al. (2005). We derive the X-ray luminosity function again from the [OIII] luminosity function of Hao et al. by combining it with the $L_{X}-L_{[\mathrm{OIII}]}$ relation derived from XMM-Newton observations (Akylas \& Georgantopoulos 2009) of all the Seyferts in the Ho et al. sample. Our analysis involves the following improvements:

- the available XMM-Newton spectra allow the correction of the X-ray absorption;

- we produce individually the luminosity function of Seyfert-1 and Seyfert-2 galaxies;

- we obtain the X-ray luminosity function from the bi-variate optical/X-ray luminosity function taking fully into consideration the $L_{\mathrm{X}}-L_{[\mathrm{OIII}]}$ relation and its dispersion.

We adopt $H_{0}=75 \mathrm{~km} \mathrm{~s}^{-1} \mathrm{Mpc}^{-1}, \Omega_{\mathrm{M}}=0.3, \Omega_{\Lambda}=0.7$ throughout the paper.

\section{The relation between $\mathrm{X}$-ray and [OIII] luminosity}

We explore the relation between the X-ray and [OIII] luminosity for the optically selected Seyfert galaxies in the Palomar spectroscopic survey of nearby galaxies (Ho et al. 1995). This survey has taken high quality spectra of 486 bright $\left(B_{T}<\right.$ $12.5 \mathrm{mag})$, northern $\left(\delta>0^{\circ}\right)$ galaxies selected from the Revised Shapley- Ames Catalogue of Bright Galaxies (RSAC, Sandage \& Tammann 1979) and produced a comprehensive and homogeneous catalogue of nearby Seyfert galaxies. Akylas \& Georgantopoulos (2009) present XMM-Newton X-ray spectra for all sources with a reliable Seyfert classification. There are 38 sources in the sample, of which 30 are classified as type2 and eight as type-1. We correct the X-ray luminosities for absorption using the observed column densities given in that paper. There are five Seyfert-2 galaxies which do not show absorption in their individual spectra. However, their stacked spectrum shows evidence for absorption. We choose to discard these galaxies from further analysis as we cannot correct accurately for their absorbed luminosity. We further discard two objects as they either have an uncertain [OIII] flux or their AGN classification has been questioned (see Akylas \& Georgantopoulos 2009). This leaves us with a sample of 23 Seyfert- 2 galaxies. A large number (12) of these show no intrinsic absorption, but still their $L_{\mathrm{X}}-L_{[\mathrm{OIII}}$ ratio is substantially lower than that of the absorbed Seyfert-2. Hereafter, we call this sub-sample the "X-ray weak" Seyfert-2 sample, while the remaining Seyfert-2 galaxies form the "X-ray luminous" sample. All eleven objects in the latter sample have very high obscuring column densities $\left(>10^{23} \mathrm{~cm}^{-2}\right)$ with the exception of NGC 4395, which has $N_{\mathrm{H}} \approx 2 \times 10^{22} \mathrm{~cm}^{-2}$. As we have only a limited number of Seyfert-1 galaxies, we expand our sample using the 20 Seyfert- 1 galaxies in the optically selected sample of Heckman et al., which is compiled from the literature (Whittle 1992; Xu et al. 1999). The X-ray luminosity of the Seyfert-1 sample of Heckman et al. (2005) is not corrected for absorption as the column densities in these sources are expected to be negligible. The [OIII] fluxes have also not been corrected for reddening.

We fit a linear model to the $\log L_{\mathrm{X}}-\log L_{[\mathrm{OIII}]}$ relation assuming no errors in $L_{X}$. The least square fits are given in Table 1 . The $L_{\mathrm{X}}-L_{\text {[OIII] }}$ relation separately for type- 1 and type- 2 sources is shown in Fig. 1. The best fit slope is below unity in all cases. This implies that the most luminous AGN have relatively little X-ray emission. Panessa et al. (2006) have also derived the $L_{X}-L_{[O I I I]}$ relation using a sample of 47 optically selected nearby AGN. These authors find a steeper than linear relation with a slope of $1.22 \pm 0.12$. Their derived slopes for the type- 1 and type- 2 slopes are $0.74 \pm 0.21$ and $1.28 \pm 0.14$ respectively. However, the comparison with their sample may not be straightforward as it includes LINERS and transient objects.

Finally, as a comparison, we quote the $L_{\mathrm{X}}-L_{[\mathrm{OIII}]}$ relation for the X-ray selected RXTE sample of Sazonov \& Revnivtsev (2004). This sample comprises 76 Seyferts detected in the 3-20 keV band down to a flux limit of $\sim 10^{-11} \mathrm{erg} \mathrm{cm}^{-2} \mathrm{~s}^{-1}$. The [OIII] luminosities have been compiled by Heckman et al. (2005) for this sample. The X-ray luminosities have been converted to the $2-10 \mathrm{keV}$ band using a photon index of $\Gamma=1.65$ (Sazonov et al. 2008) with no correction for absorption. The least square fits are given in Table 2. Interestingly, the Seyfert-1 follow a linear relation, while the Seyfert- 2 have a slope well below unity. The differences in the $L_{\mathrm{X}}-L_{[\mathrm{OIII}]}$ relation between the various samples certainly reflects the uncertainties involved in the determination of the predicted X-ray luminosity function. 
Table 2. Log X-ray vs. Log [OIII] luminosity relation in RXTE X-ray selected AGN.

\begin{tabular}{lccccc}
\hline \hline Sample & Number & Slope & Intercept & $\left\langle\log \left(L_{\mathrm{x}} / L_{[\mathrm{OIII}]}\right)\right\rangle$ & Dispersion \\
\hline Seyfert-1 & 34 & $1.03 \pm 0.12$ & $0.72 \pm 5.02$ & 1.98 & 0.39 \\
Seyfert-2 & 13 & $0.52 \pm 0.14$ & $21.41 \pm 5.74$ & 2.06 & 0.77 \\
All Seyfert & 47 & $0.88 \pm 0.04$ & $2.96 \pm 3.78$ & 2.00 & 0.51 \\
\hline
\end{tabular}
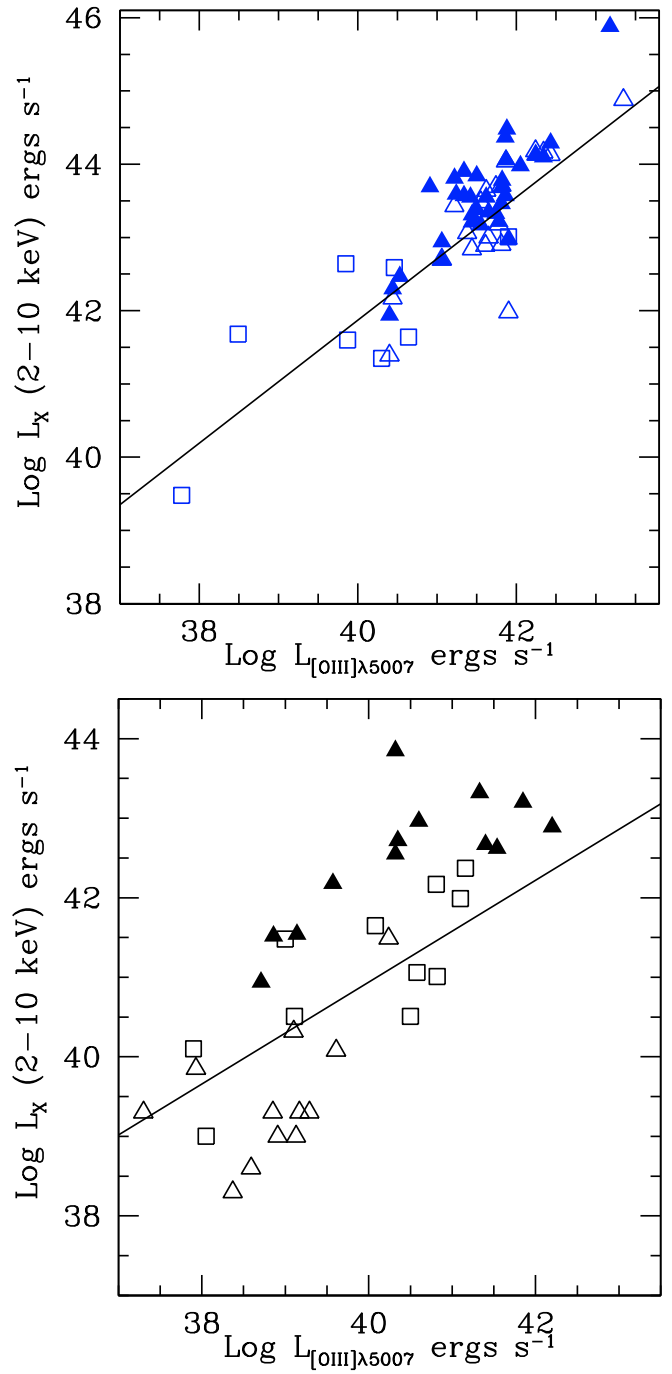

Fig. 1. Upper panel. Seyfert-1: open squares and open triangles denote sources from the samples of Ho et al. (sample B) and Heckman et al. (sample C) respectively. Filled triangles denote the data from the sample of Sazonov \& Revnivtsev (2004). The solid line is the best fit result to the joint Heckman + Ho sample (sample A). Lower panel. Seyfert-2: the open triangles refer to the "X-ray weak" (unabsorbed) Seyfert-2 (sample F), while the open squares refer to the X-ray luminous Seyfert-2s (sample E). The filled triangles denote the X-ray selected sample of Sazonov \& Revnivtsev (2004). The solid line denotes the fit to the Xray luminous Seyfert-2 (sample E).

\section{Comparison between the X-ray and the optical luminosity function}

\subsection{The [OIII] luminosity function}

Hao et al. (2005a) have obtained an AGN sample using spectroscopic SDSS data. They are using a low redshift sample $(z<0.33)$ complete down to $r=17.77$ mag over
$1151 \mathrm{deg}^{2}$. They identify broad-line AGN as those having $F W H M(\mathrm{H} \alpha)>1200 \mathrm{~km} \mathrm{~s}^{-1}$. The narrow-line AGN are selected through emission-line ratio diagnostic diagrams. Hao et al. (2005a) used both the diagnostic diagrams of Kauffman et al. (2003) and those of Kewley et al. (2001). The former criteria produce a sample which is three times larger but it is more prone to contamination from other low-luminosity AGN such as transient objects. The broad-line (Seyfert-1) and narrow-line (Seyfert-2) sample consist of 1317 and 3074 sources respectively. Their [OIII] luminosities span about three orders of magnitude $\left(10^{5}-4 \times 10^{8} L_{\odot}\right)$. Hao et al. (2005b) have parametrised the [OIII] luminosity function with a Schechter, a double powerlaw as well as a single power-law function. The single power-law function provides a very good fit to the data. For simplicity we use this functional form thereafter. We note that recently Reyes et al. (2008) extended this work to higher luminosities by constructing a catalogue of 887 type- 2 quasars from the SDSS. Their luminosity function at $z<0.3$ probes luminosities up to $10^{9} L_{\odot}$, agreeing with the extrapolation of the Hao et al. (2005b) luminosity function.

\subsection{The predicted $X$-ray luminosity function}

Having a relation between the X-ray and the optical luminosity, as well as a functional form for the optical luminosity function, we can construct the bi-variate optical/X-ray luminosity function (e.g. Georgantopoulos et al. 1999). This is given by the convolution of the optical luminosity function with a probability function which defines the value of the X-ray luminosity at a given optical luminosity. Then the X-ray luminosity function is the integral of the bi-variate luminosity function over optical luminosity.

$\Phi\left(L_{\mathrm{X}}\right)=\int \Phi\left(L_{0}\right)(1 / \sigma \sqrt{(2 \pi)}) \mathrm{e}^{-\left(L_{\mathrm{X}}-\left\langle L_{\mathrm{X}}\right\rangle\right)^{2} / 2 \sigma^{2}} \mathrm{~d} L_{0}$

where $\left\langle L_{\mathrm{X}}\right\rangle=\alpha L_{0}+\beta$ with $\alpha$ and $\beta$ given in Table 1 . The dispersion $\sigma$ plays a crucial role in the determination of the luminosity function. Large values of $\sigma$ result in higher numbers of X-ray sources in the bright X-ray luminosity bins. In order to be able to compare our results, we also calculate the predicted X-ray luminosity function for the extreme case of no dispersion i.e. $\sigma=0$. The predicted X-ray luminosity functions have been constructed as follows.

- Seyfert-1. We use the optical Seyfert-1 luminosity function of Hao et al. in combination with the $L_{X}-L_{[\mathrm{OIII}]}$ relation (samples A and C) in Table 1.

- Seyfert-2. We use the Seyfert-2 optical luminosity function of Hao et al., which is based on the Kewley criteria. We use the $L_{\mathrm{X}}-L_{[\mathrm{OIII}]}$ relation based on sample $\mathrm{E}$ (i.e. excluding the $\mathrm{X}$-ray weak Seyferts). Moreover, as a comparison we use the relation of Heckman et al. (2005) i.e. $\left\langle\log \left(L_{\mathrm{x}} / L_{[\mathrm{OIII}]}\right)\right\rangle=0.57$ with a dispersion of $\sigma=1.06$. Note that this relation has not been corrected for X-ray absorption. 
- Seyfert1+2. For the total sample we use the Seyfert luminosity function of Hao et al. combined with the $L_{X}-L_{[\mathrm{OIII}]}$ relation derived from sample $\mathrm{G}$ (see Table 1 ).

\subsection{Comparison with the observed $X$-ray luminosity function}

The X-ray luminosity has been derived in the local Universe using the RXTE slew survey data (Sazonov \& Revnivtsev 2004). The authors of this survey, construct a sample of 76 non-blazar AGN, with luminosity $L_{\mathrm{x}}>10^{41} \mathrm{erg} \mathrm{s}^{-1}$, most of them populating low redshifts $z<0.1$. They derive the luminosity function for the total sample as well as separately for the unabsorbed sample having column densities $N_{\mathrm{H}}<10^{22} \mathrm{~cm}^{-2}$. There are 59 unabsorbed sources (type-1) forming the vast majority of the sample. We note that 60 sources are classified as Seyfert-1 on the basis of their optical classification and thus there is good agreement between the X-ray and optical type- 1 classification. The total luminosity function is described by a double power-law form with a break luminosity of $L(3-20 \mathrm{keV}) \approx 4 \times 10^{43} \mathrm{erg} \mathrm{s}^{-1}$. Shinozaki et al. (2006) have derived the luminosity function of 49 AGN from the HEAO-1 all-sky survey in the $2-10 \mathrm{keV}$ band, down to a flux limit of $2.7 \times 10^{-11} \mathrm{erg} \mathrm{cm}^{-2} \mathrm{~s}^{-1}$. These authors choose to use a column density of $N_{\mathrm{H}}=10^{21.5} \mathrm{~cm}^{-2}$, as a dividing line between type- 1 and type- 2 AGN.

In Fig. 2 we compare our predicted luminosity function with the X-ray luminosity functions described above. The total predicted luminosity function $(\mathrm{Sy} 1+2)$ agrees quite well both with the luminosity function of Shinozaki et al. (2006) and of Sazonov et al. (2004). As a comparison, we also plot the luminosity function of Ueda et al. (2003) at $z=0$ as well as the INTEGRAL luminosity function derived by Sazonov et al. (2007) in the $17-60 \mathrm{keV}$ band. For the conversion to the $2-10 \mathrm{keV}$ band luminosity we assumed a slope of $\Gamma=1.65$ (Sazonov et al. 2008). It is instructive to examine the relative fraction of Seyfert1 and Seyfert-2 in the optical samples compared with the one in the X-ray selected samples. In the latter, there is a preponderance of Seyfert-1 (about 3.3:1 in RXTE). In contrast, integration of the optical luminosity functions of Seyfert-1 and Seyfert-2 shows that the two populations have approximately the same density. Only in the brighter regime $\left(\log L_{[\mathrm{OIII}]}=40.2-42.14\right)$, where there is an overlap with the X-ray luminosity function, the number of Seyfert-1 is 2.5 times higher than that of Seyfert-2 (rather more in line with the X-ray selected samples).

In a next step, we compare the luminosity functions for type- 1 and 2 Seyferts separately. We have to bear in mind that the optical luminosity function (and thus the predicted X-ray luminosity function) is based on optical line classification, while the observed X-ray luminosity function is based on X-ray spectroscopy. Nevertheless, as we saw above, the correspondence between optical and X-ray classification is reasonably good. The predicted Seyfert-1 luminosity function is somewhat higher than the observed one in the case of the $L_{X}-L_{[\mathrm{OIII}}$ relation (sample A). When we use the $L_{X}-L_{[\mathrm{OIII}]}$ relation obtained from sample $C$ instead the agreement is better. In the case of the Seyfert-2 sample the agreement between the optical and X-ray luminosity function is quite reasonable. In the Seyfert-2 samples it is possible that a low $L_{X}-L_{[\mathrm{OIII}]}$ ratio may be suggestive of excess absorption above what is directly seen in the X-ray spectra (e.g. Melendez et al. 2008; LaMassa et al. 2009). This may indeed hold true in some of the "X-ray weak" Seyfert-2 (sample F). However, this does not appear to be the case among the "luminous" Seyfert-2 (sample E) that we are using for the derivation of the Seyfert-2 luminosity function. All these Seyferts show large
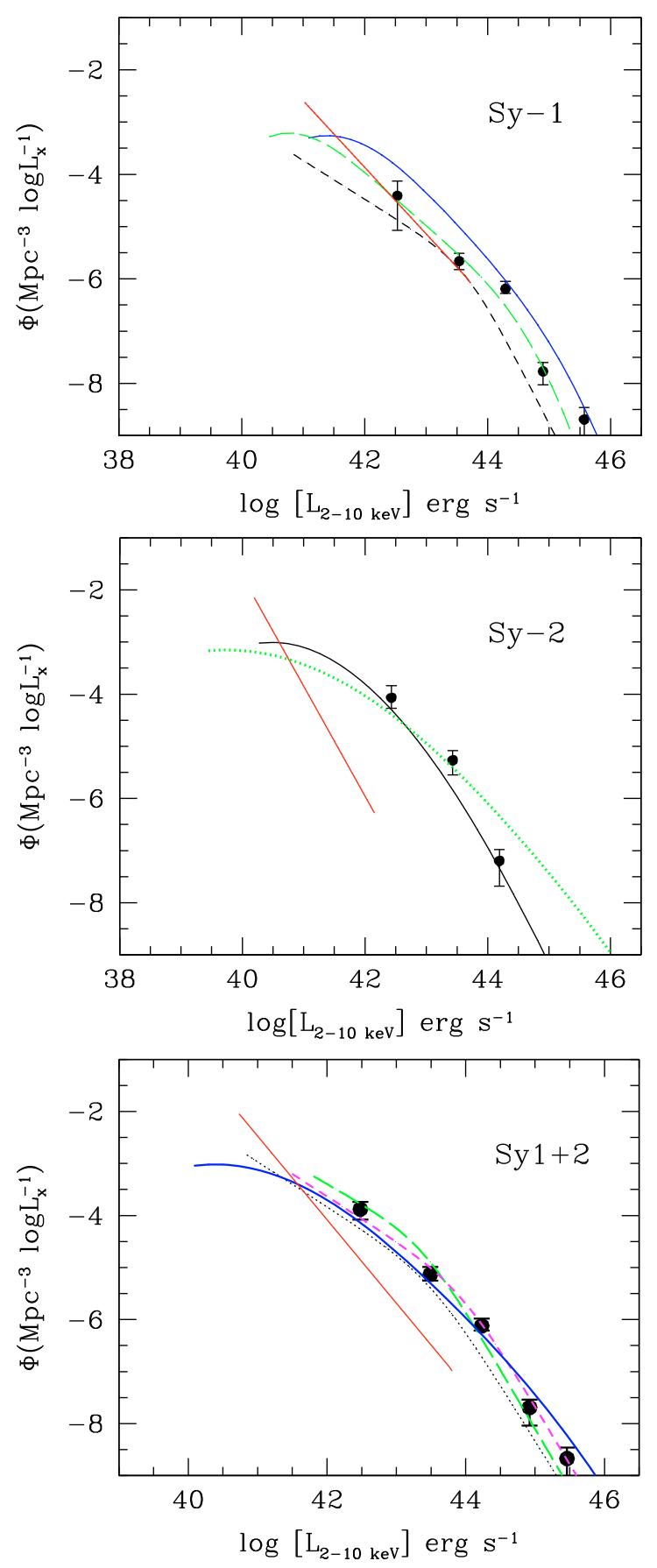

Fig. 2. Upper panel. Seyfert-1: the black points denote the HEAO1 X-ray luminosity function of Shinozaki et al. (2006); the black (short-dash) line denotes the RXTE luminosity function of Sazonov \& Revnitsev (2004); the blue (solid) line and green (long-dash) lines correspond to the predicted X-ray luminosity function for $L_{\mathrm{X}}-L_{[\mathrm{OIII}]}$ models $\mathrm{A}$ and $\mathrm{C}$ respectively. The red (solid) straight lines in all panels denote the luminosity function in the case of no dispersion in the $L_{\mathrm{X}}-L_{[\mathrm{OIII}]}$ relation $(\sigma=0)$. Seyfert-2: the black points denote the HEAO-1 X-ray luminosity function of Shinozaki et al. (2006); the red (solid) line denotes the predicted luminosity function for model $\mathrm{E}$, while the green (dot) line denotes the prediction based on the Heckman results i.e. $\left\langle\log \left(L_{x} / L_{[\mathrm{OIII}]}\right)\right\rangle=0.57$ and a dispersion of $\sigma=1.06$. Lower panel: Seyfert $1+2$. The black points denote the HEAO-1 luminosity function, while the black (dot) line denotes the RXTE luminosity function for all Seyfert galaxies; the blue (solid) line corresponds to the predicted luminosity function (model G); the green (long-dash) and purple (shortdash) curves correspond to the INTEGRAL and Chandra luminosity functions (see text for details). 
obscuring columns, and thus the correction to the intrinsic luminosity has been straightfoward.

\section{Discussion and summary}

We derived the predicted X-ray luminosity function for Seyfert galaxies in the local Universe by combining the optical SDSS [OIII] Seyfert luminosity functions with the corresponding $L_{X}-$ $L_{\text {[OIII] }}$ relation. These relations have been derived using the XMM-Newton observations (Akylas \& Georgantopoulos 2009) of the local, optically selected AGN sample of Ho et al. (1997). This sample covers a comparable luminosity range with the SDSS Seyfert sample. We have corrected the X-ray luminosity for the effects of absorption. Our analysis above shows that the predicted X-ray luminosity function is in reasonable agreement with the observed X-ray Seyfert luminosity functions obtained in the $2-10 \mathrm{keV}$ and $2-20 \mathrm{keV}$ bands by HEAO-1 and $R X T E$ respectively. Most importantly, it is in agreement with the ultra-hard 17-60 keV INTEGRAL luminosity function (Sazonov et al. 2007). As the INTEGRAL luminosity function is practically immune to X-ray absorption, this suggests that absorption played only a small role in the optical/X-ray luminosity function discrepancy reported by Heckman et al. (2005). This is also independently supported by the XMM-Newton observations of the Palomar optically selected Seyfert sample (Akylas $\&$ Georgantopoulos 2009). The fraction of Compton-thick AGN in this optically selected sample is small.

In addition we separately examined, the Seyfert- 1 and Seyfert-2 luminosity function. The Seyfert-1 luminosity function is in rough agreement with or even somewhat above the observed X-ray luminosity function. The predicted Seyfert-2 luminosity function agrees quite well with the optical luminosity function again disfavouring the absorption hypothesis. Indeed, if absorption were the problem, then our predicted luminosity function, which takes X-ray absorption into account, would be well above that of RXTE.

Having addressed this matter, we need to understand why Heckman et al. (2005) found that the RXTE X-ray luminosity function lies below the optical one by about a factor of three. The comparison was based on the fact that the two luminosity functions have the same slope. However, this is true only for the bright part of the X-ray luminosity function (i.e. for $L_{\star}(3-20 \mathrm{keV})>3 \times 10^{43} \mathrm{erg} \mathrm{s}^{-1}$ or equivalently $\left.L_{\star}(2-10 \mathrm{keV})>2.3 \times 10^{43} \mathrm{erg} \mathrm{s}^{-1}\right)$. Using $L_{\mathrm{X}} / L_{[\mathrm{OIII}]} \approx 100$, the corresponding optical luminosities must be greater than $5.7 \times 10^{7} L_{\odot}$. Since the upper end of the optical luminosity function of Hao et al. (2005b) is $\sim 3 \times 10^{8} L_{\odot}$, the overlap is very limited (see Fig. 5 in Heckman et al.), and thus the comparison is not quite robust. Moreover, the effect of the dispersion plays a critical role, as shown here.

Although the densities of the [OIII] and the X-ray selected AGN are comparable, this does not mean that these methods favour the selection of the same objects. The optical selection favours X-ray weak Seyfert-2 (see Fig. 1). It has been proposed (see Ho 2008, for a review) that a large number of sources at low luminosities appear as Seyfert-2 possibly because no broadline-region is formed at low accretion rates (Nicastro 2000) or low luminosities (Elitzur \& Shlosman 2006). If the above models hold true, these "naked" Seyfert-2 galaxies should present no hidden broad lines in spectropolarimetric observations (e.g. Tran 2003). Interestingly, Spinoglio et al. (2009) find that the ratio of the X-ray to $12 \mu \mathrm{m}$ luminosity, $L_{\mathrm{X}} / L_{12 \mu \mathrm{m}}$, is much lower in the Seyfert- 2 with no hidden broad-line-region, suggesting that these are weak X-ray emitters. There is however one caveat in these "naked" X-ray weak Seyfert-2 interpretation. The [OIII] (or the mid-IR) luminosity is believed to be a good proxy of the ionizing nuclear luminosity and thus to the X-ray luminosity. But in these Seyfert-2 sources the $L_{\mathrm{X}} / L_{[\mathrm{OIII}]}$ ratio is low, implying that only the X-ray luminosity is weak. One explanation could be that star-formation is contributing a large part of the [OIII] emission.

The future missions NUSTAR and ASTRO-H, having superb imaging capabilities $(\sim 1$ arcmin) at ultra-hard energies (10-70 keV), will provide the opportunity to obtain the least unbiased AGN samples, reaching flux levels of at least two orders of magnitude fainter than SWIFT and INTEGRAL.

\section{References}

Akylas, A., \& Georgantopoulos, I. 2009, A\&A, 500, 999 Alexander, D. M., Bauer, F. E., Brandt, W. N., et al. 2003, AJ, 126, 539 Barger, A. J., Cowie, L. L., Capak, P., et al. 2003, AJ, 126, 632 Barger, A. J., Cowie, L. L., Mushotzky, R. F., et al. 2005, AJ, 129, 578 Bassani, L., Molina, M., Malizia, A., et al. 2006, ApJ, 636, L65 Bauer, F. E., Alexander, D. M., Brandt, W. N., et al. 2004, AJ, 128, 2048 Beckmann, V., Gehrels, N., Shrader, C. R., \& Soldi, S. 2006, ApJ, 638, 642 Boyle, B. J., Shanks, T., Croom, S. M., et al. 2000, MNRAS, 317, 1014 Brandt W. N., \& Hasinger, G. 2005, ARA\&A, 43, 827

Cappi, M., Panessa, F., Bassani, L., et al. 2006, A\&A, 446, 459 Comastri, A. 2004, ASSL, 308, 245

Croom, S. M., Smith, R. J., Boyle, B. J., et al. 2004, MNRAS, 349, 1397 Elitzur, M., \& Shlosman, I. 2006, ApJ, 648, L101

Giacconi, R., Zirm, A., Wang, J., et al. 2002, ApJS, 139, 369

Gilli, R., Comastri, A., \& Hasinger, G. 2007, A\&A, 463, 79

Hao, L., Strauss, M. A., Tremonti, C. A., et al. 2005a, AJ, 129, 1783

Hao, L., Strauss, M. A., Fan, X., et al. 2005b, AJ, 129, 1795

Heckman, T. M., Ptak, A., Hornschemeier, A., \& Kauffmann, G. 2005, ApJ, 634, 161

Ho, L. C. 2008, ARA\&A, 46, 475

Kauffmann, G., Heckman, T. M., Tremonti, C., et al. 2003, MNRAS, 346, 1055 Kellogg, E., Gursky, H., Leong, C., et al. 1971, ApJ, 165, L49

La Franca, F., Fiore, F., Comastri, A., et al. 2005, A\&A, 635, 864

LaMassa, S., Heckman, T. M., Ptak, A. A., et al. 2009 [arXiv: 0909. 3044]

Luo, B., Bauer, F. E., Brandt, W. N., et al. 2008, ApJS, 179, 19

Marshall, H. 1987, AJ, 94, 628

Matt, G., Fabian, A. C., Guainazzi, M., et al. 2000, MNRAS, 318, 173

Melendez, M., Kraemer, S. B., Armentrout, B. K., et al. 2008, ApJ, 682, 94

Nicastro, F. 2000, ApJ, 530, L65

Paltani, S., Walter, R., McHardy, I. M., et al. 2008, A\&A, 485, 707

Risaliti, G., Maiolino, R., \& Salvati, M. 1999, ApJ, 522, 157

Sandage, A. R., \& Tammann, G. A. 1981, A revised Shapley-Ames catalog of Bright Galaxies, Carnegie Inst. Washington

Sazonov, S. Y., \& Revnivtsev, M. G. 2004, A\&A, 423, 469

Sazonov, S., Revnivtsev, M., Krivonos, R., Churazov, E., \& Sunyaev, R. 2007, A\&A, 462, 57

Sazonov, S., Krivonos, R., Revnivtsev, M., Churazov, E., \& Sunyaev, R. 2008, A\&A, 482, 517

Schmidt, M., \& Green, R. F. 1983, ApJ, 269, 352

Shinozaki, K., Miyaji, T., Ishisaki, Y., Ueda, Y., \& Ogasaka, Y. 2006, AJ, 131, 2843

Spinoglio et al. 2009 [arXiv:0901.1212]

Tran, H. D. 2003, ApJ, 583, 632

Treister, E., Urry, C. M., \& Virani, S. 2009, ApJ, 696, 110

Tueller, J., Mushotzky, R. F., Barthelmy, S., et al. 2009, ApJ, 681, 113

Ueda, Y., Akiyama, M., Ohta, K., \& Miyaji, T. 2003, ApJ, 598, 886

Ulvestad, J. S., \& Ho, L. C. 2001, ApJ, 558, 561

Werner, M. W., Roellig, T. L., \& Low, F. J. 2004, ApJS, 154, 1

Whittle, M. 1992, ApJS, 79, 49

Winkler, C., Courvoisier, T. J.-L., Di Cocco, G., et al. 2003, A\&A, 411, L1

Winter, L. M., Mushotzky, R. F., Tueller, J., \& Markwardt, C. 2008, ApJ, 674, 686

Winter, L. M., Mushotzky, R. F., Reynolds, C. S., \& Tueller, J. 2009, ApJ, 690, 1322

Wolf, C., Wisotzki, L., Borch, A., et al. 2003, A\&A, 408, 499

Xu, C., Livio, M., \& Baum, S. 1999, AJ, 118, 1169

York, D. G., Adelman, J., \& Anderson, J. E. 2000, AJ, 120, 1579 